\title{
Highly efficient optical power transfer to whispering-gallery modes by use of a symmetrical dual-coupling configuration
}

\author{
Ming Cai and Kerry Vahala \\ Department of Applied Physics, California Institute of Technology, Pasadena, California 91125
}

Received September 28, 1999

\begin{abstract}
We report that greater than $99.8 \%$ optical power transfer to whispering-gallery modes was achieved in fusedsilica microspheres by use of a dual-tapered-fiber coupling method. The intrinsic cavity loss and the taper-tosphere coupling coefficient are inferred from the experimental data. It is shown that the low intrinsic cavity loss and the symmetrical dual-coupling structure are crucial for obtaining the high coupling efficiency. (c) 2000 Optical Society of America

OCIS codes: $230.5750,230.3990,060.1810,230.1150$.
\end{abstract}

Whispering-gallery mode (WGM) resonances in dielectric microspheres are receiving considerable attention in both fundamental and applied studies. ${ }^{1-3}$ Many coupling techniques, such as prisms, ${ }^{4}$ half-block couplers,${ }^{5}$ tapered fibers, ${ }^{2,6}$ and angle-polished fiber couplers, ${ }^{7}$ have been demonstrated. In our recent work we demonstrated that 99\% power transfer was achievable with a novel dual-tapered-fiber coupling structure. $^{2}$ In this Letter we investigate the symmetrical dual-coupling scheme in greater detail and for various-sized cavities to explain its excellent coupling performance. It is demonstrated that more than $99.8 \%$ of the incident optical power can be transferred into a whispering-gallery resonant mode in a fusedsilica microsphere. A simple model is used to simulate the interaction of the electromagnetic field in the couplers and the microsphere. The analysis shows that it is possible to achieve full transfer of the optical power to certain WGM's (critical coupling) by control of the coupling coefficient and the cavity loss. The symmetrical dual-coupling structure can permit the maintenance of the critical coupling condition for a wide range of modes.

A schematic of the dual-coupling WGM resonant system is shown in Fig. 1(a). Two nearly identical taper couplers are placed tangentially at the opposite sides of the equator of a microsphere. One serves as the input coupler, and the other is the output coupler. Light at a resonant wavelength that is launched into the input coupler will be coupled into the cavity and will build up a large circulating optical power. A small fraction of this circulating power is reemitted to the input coupler, where it joins the field that is directly transmitted past the sphere (i.e., the uncoupled field) and forms the net transmitted field $E_{t}$. Likewise, at the other taper a small portion of the circulating power is coupled out to the second fiber taper as $E_{k}$. This coupling effectively acts as an added cavity loss in the dual-taper coupling measurements. Figure 1(b) is a magnified photograph of our actual system and shows two tapered fibers attached to a fused-silica microsphere. The shadow on the background is the image of the glass stem that is used to support and manipulate the microsphere. The fiber tapers had a waist of $\sim 3 \mu \mathrm{m}$. They were made by use of the technique described in Refs. 2 and 6.

To characterize the system we measured the transmission spectra at the throughput port of the input fiber. For comparison, we measured the transmission spectra first in the dual-taper and then in the single-taper coupling configuration. We did this by first measuring the dual-taper system spectra and then gently detaching the second fiber taper from the sphere to maintain the coupling condition between the first fiber taper and the microsphere. Data on loss and coupling (see below) confirmed that the first fiber taper coupling was not significantly affected by the detachment procedure. A tunable external-cavity diode laser with a linewidth of less than $300 \mathrm{kHz}$ was

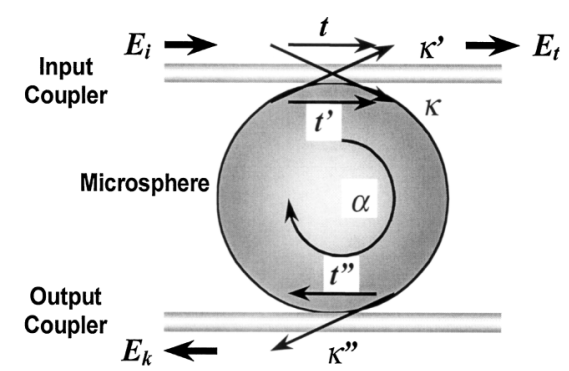

(a)

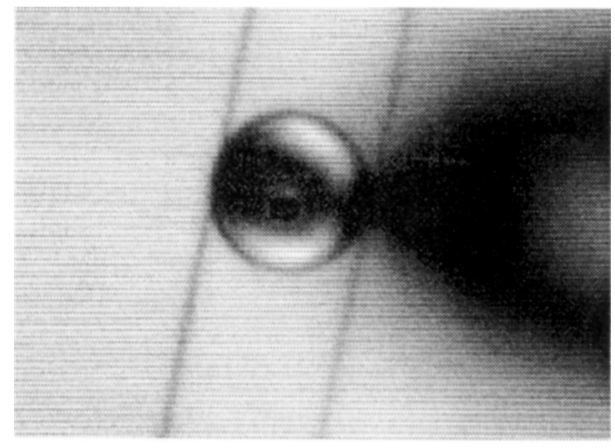

(b)

Fig. 1. (a) Schematic of a WGM resonator in the dualtaper coupling configuration. (b) Magnified photograph of the actual system. The background shadow in (b) is produced by the fiber stem that is used to hold the sphere. 
used as the laser source. The laser was continuously scanned near $1.55 \mu \mathrm{m}$. Figure 2 shows the normalized transmission spectra for a microsphere with a diameter of $\sim 140 \mu \mathrm{m}$. Figure 2(a) is the dual-coupling case, and Fig. 2(b) is the corresponding single-coupling case. The transmission dips observed in the spectra correspond to a series of WGM resonances at different wavelengths. The measured transmitted power at the center frequency of the resonance marked with an asterisk in Fig. 2(a) was less than $-28 \mathrm{~dB}$, corresponding to a power transfer efficiency greater than $99.8 \%$. For those resonances marked with arrows the transmittance was less than $-20 \mathrm{~dB}$, corresponding to greater than $99 \%$ power transfer. Note that in our singlecoupling case the coupling efficiencies are generally in the range $40-80 \%$, which are comparable with published results for the single-coupling scheme. ${ }^{6}$

We repeated the same experiments for various-sized spheres in a range from 300 to $30 \mu \mathrm{m}$ in diameter. Greater than $99 \%$ power transfer was routinely observed in the dual-coupling configuration. Using a microsphere with a diameter of $\sim 34 \mu \mathrm{m}$, we measured the linewidth of a resonance to be $15 \mathrm{GHz}$ in the dual-coupling condition. The coupling efficiency, however, remained as high as $99.3 \%$. The resonantly transferred power to the second fiber was $\sim 85 \%$ of the power transmitted through the first fiber in the nonresonant condition. This result shows the potential of the dual-coupling configuration as an add-drop filter for telecommunication applications in which high extinction, high drop efficiency, and a bandwidth of several gigaghertz are required.

To understand the highly efficient power transfer in our experiments we used a simple model (similar to the model in Ref. 8, except that two couplers were considered in our case) to describe the system. As shown in Fig. 1(a), $\kappa$ is the amplitude of the field-coupling coefficient from the input coupler to the sphere, and $t$ is the amplitude of the fieldtransmission coefficient through the coupler from the region before coupling to after the coupling. Similarly, $\left(\kappa^{\prime}, \kappa^{\prime \prime}\right)$ and $\left(t^{\prime}, t^{\prime \prime}\right)$ are the corresponding amplitudes of the coupling coefficients from the sphere to the couplers and the transmission coefficients inside the sphere, respectively. Subscripts 1 and 2 will be used hereafter to refer to the quantities in the singleand dual-coupling cases, respectively. $\alpha$ is the amplitude loss of the cavity per round trip (caused by material absorption, whispering-gallery radiation loss, scattering from surface defects and contaminants, taper coupler scattering into radiation modes, etc.) and represents the fraction of the remaining field amplitude after one round trip in the sphere (excluding the taper coupling). Assuming that the power scattering into radiation modes at the coupling region is negligible, ${ }^{9}$ we can express the power transmittance on resonance:

$$
T_{1,2}=\left|\frac{E_{t}}{E_{i}}\right|^{2}=\left|\frac{t_{1,2}-\alpha_{1,2}}{1-\alpha_{1,2} t_{1,2}}\right|^{2}
$$

where $\alpha_{1}$, the total cavity loss in the single-coupling case, is simply equal to $\alpha$ and $\alpha_{2}$, the total cavity loss in the dual-coupling case, is equal to $\alpha t^{\prime \prime}$. It is clear that $t_{1}=\alpha_{1}\left(t_{2}=\alpha_{2}\right)$ is required if one is to achieve zero transmittance for single-taper coupling (dual-taper coupling). This condition is referred to as critical coupling. The quality factor of a loaded resonant mode is defined by $Q=\omega / \Delta \omega$, where $\Delta \omega$ is the full width of the resonance at the half-power points. From the model, one can derive $\Delta \omega$, and thus the quality factor,

$$
Q_{1,2}=\frac{\omega}{\Delta \omega}=\frac{2 \pi^{2} n R}{\lambda} \frac{\sqrt{\alpha_{1,2} t_{1,2}}}{1-\alpha_{1,2} t_{1,2}},
$$

where $R$ is the radius of the sphere, $n$ is the refractive index, and $\lambda$ is the resonant wavelength. With Eqs. (1) and (2), the transmission coefficient $t_{1,2}$ and the total cavity loss $\alpha_{1,2}$ for each resonant mode can be inferred from the experimental data of the transmittance and the quality factor. Generally, it is more interesting to specify the coupling coefficient $\kappa_{1}\left(\kappa_{2}\right)$

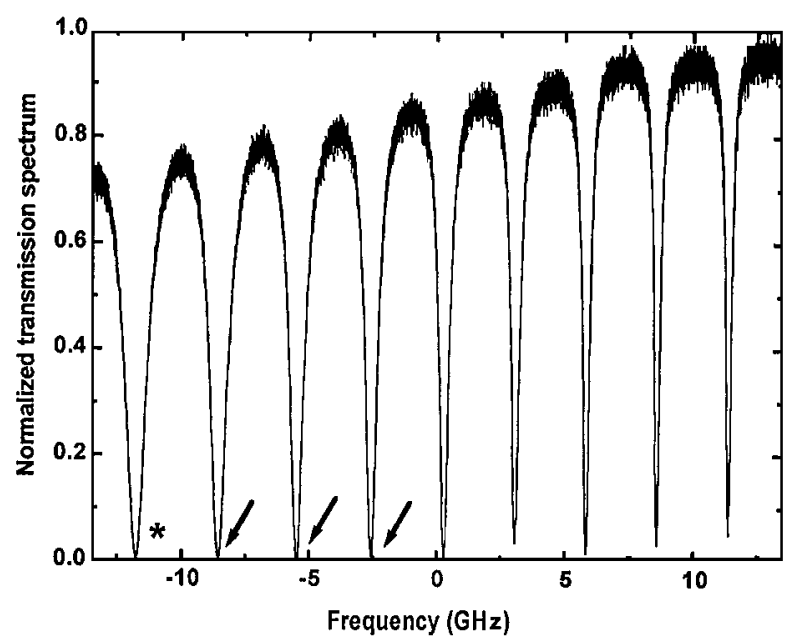

(a)

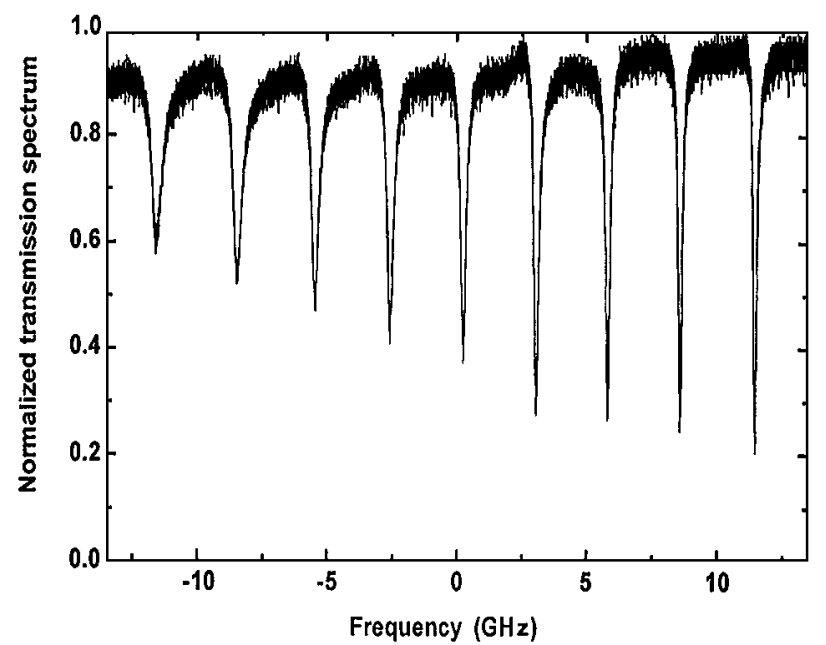

(b)

Fig. 2. Normalized transmission spectra for the (a) dualtaper coupling configuration and (b) single-taper coupling configuration. The resonance marked with an asterisk has a power transfer efficiency greater than $99.8 \%$. Those marked with arrows have power tranfser efficiencies in excess of $99 \%$. The sphere used in these measurements is $\sim 140 \mu \mathrm{m}$ in diameter. 


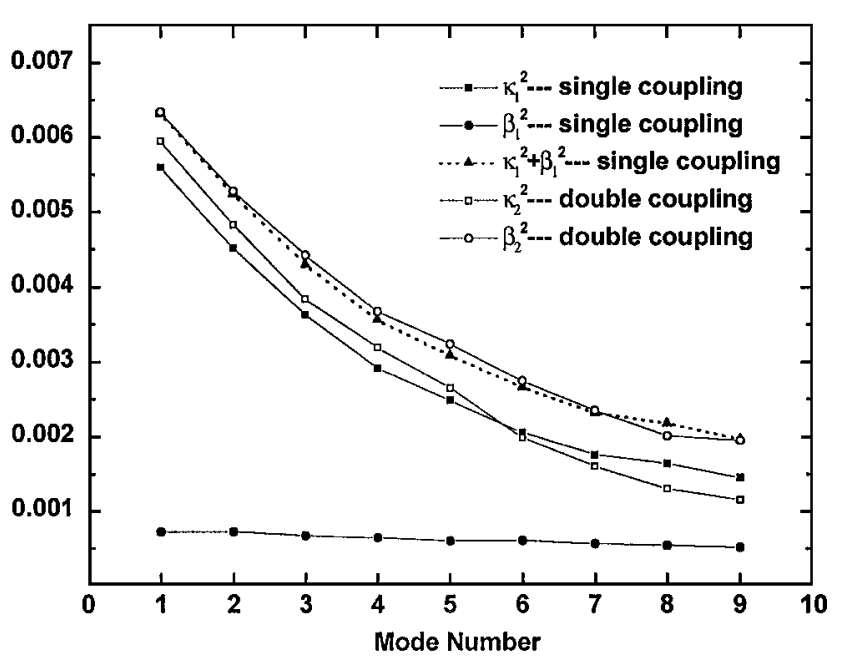

Fig. 3. Calculated coupling coefficients $\kappa_{1}{ }^{2}, \kappa_{2}{ }^{2}$ and total cavity loss $\beta_{1}{ }^{2}, \beta_{2}{ }^{2}$, corresponding to the spectra shown in Fig. 2. $\kappa_{1}{ }^{2}+\beta_{1}{ }^{2}$ and $\beta_{2}{ }^{2}$ are in close agreement, showing that the second coupler is nearly identical to the first coupler.

rather than the transmission coefficient $t_{1}\left(t_{2}\right)$. Also, for convenience, we define $\beta_{1}\left(\beta_{2}\right)$, the total loss coefficients of the cavity, in terms of $\alpha_{1}\left(\alpha_{2}\right)$ as $\left(\beta_{1,2}\right)^{2}+$ $\left(\alpha_{1,2}\right)^{2}=1$.

Figure 3 shows the calculated results for $\kappa_{1}{ }^{2}\left(\kappa_{2}{ }^{2}\right)$ and $\beta_{1}{ }^{2}\left(\beta_{2}{ }^{2}\right)$, corresponding to the transmission spectra in Fig. 2. Note that $t_{1,2}$ and $\alpha_{1,2}$ (and thus $\kappa_{1,2}$ and $\beta_{1,2}$ ) are symmetrical in both Eq. (1) and Eq. (2). They can be identified, however, by comparison of the results from the single-coupling case with those from the dual-coupling case. Since the coupling condition of the input fiber is believed to remain nearly unchanged in our experiments, $\kappa_{1}{ }^{2}$ and $\kappa_{2}{ }^{2}$ should be nearly equal for each resonance in both the single- and the dualcoupling conditions. The calculated results in Fig. 3 confirm this assertion.

Ideally, the loss coefficient $\beta_{2}{ }^{2}$ in the case of dualtaper coupling will be the simple combination of $\beta_{1}{ }^{2}$ and $\kappa^{\prime \prime 2}$ (assuming that the coupling of the output fiber does not introduce any significant additional loss aside from the coupling loss $\kappa^{\prime \prime 2}$ ). We fit $\beta_{2}{ }^{2}$ by use of $\beta_{1}{ }^{2}$ and $\kappa_{1}{ }^{2}$, and the fact that ${\beta_{2}}^{2} \approx \beta_{1}{ }^{2}+\kappa_{1}{ }^{2}$ indicates that the output fiber exhibits a nearly identical coupling coefficient for each resonance as the input fiber. This fact also shows that the second coupler introduces minimal scattering loss into the radiation modes. The microsphere resonator is therefore in nearly symmetrical coupling conditions in the double-taper configuration.

The symmetrical coupling structure plays an important role in obtaining the nearly critically coupled resonances. From Figs. 2 and 3, the intrinsic loss $\left(\beta_{1}{ }^{2}\right)$ was observed to be nearly independent of the mode index and to remain very small. ${ }^{10}$ In our experiments $\beta_{1}^{2}$ was approximately three to ten times smaller than the coupling coefficients $\left(\kappa_{1}^{2}\right)$ of the observed modes in the single-taper geometry. The dualcoupling case, however, is quite different. The output coupler introduces a mode-dependent loss to the resonant modes that tends to track the coupling through the first taper. Therefore, as shown in the spectrum of the dual-coupling case [Fig. 2(a)], the critical coupling condition can be nearly satisfied for a wide range of modes. Furthermore, since most of this added loss comes about from coupling to the taper mode (not scattering), the high fiber-to-fiber coupling efficiencies observed in our previous work ${ }^{2}$ have now been explained.

In summary, we have demonstrated greater than 99.8\% optical power transfer to whispering-gallery modes based on coupling of a tapered fiber to a fusedsilica microsphere. In addition, greater than 99\% power transfer efficiency has been routinely observed in various-sized cavities. Data combined with modeling reveal that the low intrinsic loss of the cavity (including low scattering loss at the taper-sphere coupling) combined with the ability to create nearly identical dual-taper couplings are the key ingredients in this high power-transfer efficiency.

This work was supported by the Defense Advanced Research Projects Agency. The authors thank O. Painter for valuable discussions. K. J. Vahala thanks A. Yariv for helpful discussions on critical coupling. K. J. Vahala's e-mail address is vahala@its.caltech.edu.

\section{References}

1. M. L. Gorodetsky, A. A. Savchenkov, and V. S. Ilchenko, Opt. Lett. 21, 453 (1998).

2. M. Cai, G. Hunziker, and K. Vahala, IEEE Photon. Technol. Lett. 11, 686 (1999).

3. V. Sandoghdar, F. Treussart, J. Hare, V. Lefevre-Segin, J.-M. Raimond, and S. Haroche, Phys. Rev. A 54, 1777 (1996).

4. M. L. Gorodetsky and V. S. Ilchenko, Opt. Commun. 113, 133 (1994).

5. A. Serpenguzel, S. Arnold, and G. Griffel, Opt. Lett. 20, 654 (1995).

6. J. C. Knight, G. Cheung, F. Jacques, and T. A. Birks, Opt. Lett. 22, 1129 (1997).

7. V. S. Ilchenko, A. S. Yao, and L. Maleki, Opt. Lett. 24, 723 (1999).

8. G. Griffel, S. Arnold, D. Taskent, and A. Serpenguzel, Opt. Lett. 21, 695 (1995).

9. With this assumption, we approximate the coupler as nearly lossless so that reciprocity and energyconservation relations can be used to relate the various coupling coefficients. The validity of this assumption is affirmed by the experimental results.

10. Not all devices tested behaved in this manner. Scattering at the taper-sphere coupling is expected in general to be mode dependent and to provide the most significant source of non-coupling-related loss for WGM's. Scattering is believed to be the dominant source of non-coupling-related loss in the current experiments. 